\title{
Undesirable Outputs' Presence in Centralized Resource Allocation Model
}

\author{
Ghasem Tohidi, Hamed Taherzadeh, and Sara Hajiha \\ Department of Mathematics, Islamic Azad University, Central Branch, Tehran, Iran \\ Correspondence should be addressed to Hamed Taherzadeh; h.taherzadeh@hotmail.com
}

Received 15 July 2014; Revised 25 August 2014; Accepted 28 August 2014; Published 15 September 2014

Academic Editor: Vikas Kumar

Copyright (C) 2014 Ghasem Tohidi et al. This is an open access article distributed under the Creative Commons Attribution License, which permits unrestricted use, distribution, and reproduction in any medium, provided the original work is properly cited.

\begin{abstract}
Data envelopment analysis (DEA) is a common nonparametric technique to measure the relative efficiency scores of the individual homogenous decision making units (DMUs). One aspect of the DEA literature has recently been introduced as a centralized resource allocation (CRA) which aims at optimizing the combined resource consumption by all DMUs in an organization rather than considering the consumption individually through DMUs. Conventional DEA models and CRA model have been basically formulated on desirable inputs and outputs. The objective of this paper is to present new CRA models to assess the overall efficiency of a system consisting of DMUs by using directional distance function when DMUs produce desirable and undesirable outputs. This paper initially reviewed a couple of DEA approaches for measuring the efficiency scores of DMUs when some outputs are undesirable. Then, based upon these theoretical foundations, we develop the CRA model when undesirable outputs are considered in the evaluation. Finally, we apply a short numerical illustration to show how our proposed model can be applied.
\end{abstract}

\section{Introduction}

Data envelopment analysis (DEA) was introduced in 1978. DEA includes many models for assessing the efficiency score in the variety of conditions. Many researchers use this technique to evaluate the efficiency and inefficiency scores of decision making units (DMUs). Two of the most common DEA models are CCR (Charnes, Cooper, and Rhodes) and BCC (Banker, Charnes, and Cooper) which were introduced by Charnes et al. [1] and Banker et al. [2], respectively. In addition, there are other important models such as additive (ADD) model which was introduced by Charnes et al. [3] and SMB model (slack-based measure) which was introduced by Tone [4]. Classical DEA models (such as CCR, BCC, ADD, and SMB) rely on the assumption that inputs have to be minimized and outputs have to be maximized. In authentic situations, however, it is possible that the production process consumes undesirable inputs and/or generates undesirable outputs $[5,6]$. Consequently, classical DEA models need to be modified in order to deal with the situation because undesirable outputs should not maximize at all.
There frequently exist undesirable inputs and/or outputs in the real applications. Many studies have been done on the undesirable data. Broadly, we can divide these studies into two parts. The first part involves some methods, which use transformation data. For instance, Koopman [6] suggested data transformation. Although the reflection function was used in this method, it caused the positive data to turn into negative data and it was not straightforward to define efficiency score for negative data at that time. Some of the related methods had been suggested by Iqbal Ali and Seiford [7], Pastor [8], Scheel [9], and Seiford and Zhu [10]. However, Golany and Roll [11] and Lovell and Pastor [12] attempted to introduce another form of transformation, which was multiplicative inverse. Being a nonlinear transformation, its behaviors were even more complicated to deal with (Scheel [13]). Therefore, the approaches based on data transformation may unexpectedly produce unfavorable results, such as those discussed by Liu and Sharp [14]. The second part consists of many methods, which can avoid data transformation. As an initial attempt, Liu and Sharp [14] suggested considering undesirable outputs as desirable inputs but undesirable inputs as desirable outputs. This method is currently used as an 
attractive one in studying operational efficiency because of its simplicity and elegance.

In many authentic situations, there are cases in which all DMUs are under the control of a centralized decision maker (DM) that oversees them and tends to increase the efficiency of all of the system instead of increasing the efficiency of each unit separately. These situations occur when all of the units belong to the same organization (public and/or private) which provides the units with the necessary resources to obtain their outputs, such as bank branches, restaurant chains, hospitals, university departments, and schools. Thus, DM's goal is to optimize the resource utilization of all DMUs across the total entity. Lozano and Villa [15] first introduced the meaning of centralized resource allocation. They presented the envelopment and multiplier form of BCC model with regard to centralized meaning. Mar-Molinero et al. [16] demonstrated that the centralized resource allocation model proposed by Lozano and Villa [15] can be substantially simplified. There are some other similar researches done by Korhonen and Syrjänen [17], Du et al. [18], and Asmild et al. [19]. Multiple-objective model has been used in order to optimize the efficiency of system by Korhonen and Syrjänen [17], and Du et al. [18] proposed another approach for optimization in centralized scenario. Asmild et al. [19] reformulated the centralized model proposed by Lozano and Villa [15] considering adjustments of inefficient units. Hosseinzadeh Lotfi et al. [20] and Yu et al. [21] are other researchers engaged in centralized resource allocation.

In this paper we discuss a DEA model in centralized resource allocation when some of the inputs or outputs are undesirable. This paper is organized as follows. In Section 2, research motivation of this study is given. Section 3 briefly presents some methods for measuring the efficiency scores when some of the outputs are undesirable. Section 4 discusses the centralized resource allocation model and its advantages. We develop the centralized resource allocation model in the undesirable outputs' presence in Section 5. An illustration is given in Section 6 and Section 7 provides the conclusion of the paper.

\section{Research Motivation}

Traditional DEA models are consecrated to the performance evaluation of DMUs in different situations. Although undesirable outputs treatments have been studied by interested researchers, centralized resource allocation has never dealt with undesirable outputs. Moreover, in many real situations, the production of undesirable outputs is unavoidable; hence, decision makers need scientific methods to deal with the undesirable outputs' production and decrease them when all of DMUs are under their control. Here, we will answer the following question scientifically: how can centralized resource allocation model be modified in order to evaluate the performance of a system involving several DMUs which produce both desirable and undesirable outputs?

\section{Undesirable Output Models}

Most researchers recently analyze closely the structure of the undesirable data. Undesirable outputs, such as air purification, sewage treatment, and wastewater, can be jointly produced with desirable outputs. When the undesirable outputs are taken into account, the efficiency score's evaluation of DMUs is different. Therefore, traditional DEA models should be modified. Briefly, we review a couple of methods to measure the efficiency scores when some of the data are undesirable and we address some papers for evaluating undesirable data.

Seiford and Zhu [10] showed that the traditional DEA model is used to improve the performance through increasing the desirable outputs and decreasing undesirable outputs by the classification invariance property use. Their model can also be applied to a situation when inputs need to be increased to improve the performance. This model is as follows:

$$
\begin{array}{ll}
\max & \phi \\
\text { s.t. } & \lambda X \leq x_{o}^{D} \\
& \lambda Y^{D} \geq \phi y_{o}^{D} \\
& \lambda \bar{Y}^{U} \geq \phi \bar{y}_{o}^{U} \\
& e \lambda=1 \\
& \lambda \geq 0,
\end{array}
$$

in which $\bar{y}_{o}^{U}=-Y^{U}+v>0$. Hadi Vencheh et al. [22] proposed a model for treating undesirable factors in the framework of DEA as follows:

$$
\begin{array}{ll}
\max & \phi \\
\text { s.t. } & \lambda X^{D} \leq(1-\phi) x_{o}^{D} \\
& \lambda \bar{X}^{U} \leq(1-\phi) \bar{x}_{o}^{U} \\
& \lambda Y^{D} \geq(1+\phi) y_{o}^{D} \\
& \lambda \bar{Y}^{U} \geq(1+\phi) \bar{y}_{o}^{U} \\
& e \lambda=1 \\
& \lambda \geq 0,
\end{array}
$$

in which $\bar{y}_{o}^{U}=-Y^{U}+v>0$ and $\bar{X}^{U}=-X^{U}+w>0$ (Seiford and Zhu [10]). Model (2) evaluates the efficiency level of each DMU by considering desirable and undesirable factors. In fact, model (2) expands desirable outputs and contracts undesirable outputs. A similar discussion holds for the inputs. Jahanshahloo et al. [23] presented an alternative method to deal with desirable and undesirable factors (inputs and outputs) in nonradial DEA models. They demonstrated 
that their proposed model is feasible, bounded, and unit invariant. The model is given as follows:

$$
\begin{array}{ll}
\min & 1-\left[w_{o}+\frac{1}{m+s}\left(\sum_{i \in I_{D}} t_{i}^{-D}+\sum_{r \in O_{D}} t_{r}^{+D}\right)\right] \\
\text { s.t. } & \sum_{j=1}^{n} \lambda_{j} x_{i j}^{D}+t_{i}^{-D}=x_{i o}^{D}-w_{o} \quad i \in I_{D} \\
& \sum_{j=1}^{n} \lambda_{j} x_{i j}^{U}+t_{i}^{-U}=x_{i o}^{U}+w_{o} \quad i \in I_{U} \\
& \sum_{j=1}^{n} \lambda_{j} y_{r j}^{D}-t_{r}^{+D}=y_{r o}^{D}+w_{o} \quad r \in O_{D} \\
& \sum_{j=1}^{n} \lambda_{j} y_{r j}^{U}-t_{r}^{+U}=y_{r o}^{U}-w_{o} \quad r \in O_{U} \\
& \sum_{j=1}^{n} \lambda_{j}=1,
\end{array}
$$

in which all variables are restricted to be nonnegative. In model (3), $I_{D}, I_{U}, O_{D}$, and $O_{U}$ stand for desirable inputs, undesirable inputs, desirable outputs, and undesirable outputs, respectively. Recently, Wu and Guo [24] suggested a model for measuring the efficiency score which is formulated based on that inputs and undesirable outputs are decreased proportionally. This model is as follows:

$$
\begin{array}{ll}
\min & \theta \\
\text { s.t. } & \sum_{j=1}^{n} \lambda_{j} x_{i j} \leq \theta x_{i o} \quad \forall i \in I \\
& \sum_{j=1}^{n} \lambda_{j} y_{r j}^{D} \geq y_{r o}^{D} \quad \forall r \in O^{D} \\
& \sum_{j=1}^{n} \lambda_{j} y_{r j}^{U} \leq \theta y_{r o}^{U} \quad \forall r \in O^{U} \\
& \lambda_{j} \geq 0 \quad \forall j \in N .
\end{array}
$$

In model (4), $I, O^{D}$, and $O^{U}$ refer to inputs, desirable outputs, and undesirable outputs sets, respectively. The studies of Scheel [9] and Amirteimoori et al. [25] are another two studies. Indeed, Scheel [9] proposed new efficiency measures which are oriented to desirable and undesirable outputs, respectively. They are based on the assumption that any change of output levels involves both desirable and undesirable outputs. Amirteimoori et al. [25] presented a DEA model which can be used to improve the relative performance via increasing undesirable inputs and decreasing undesirable outputs.

\section{Centralized Resource Allocation Model}

Measuring the performance plays an important role for a DM providing its weaknesses for the subsequent improvement. Working on the usual DEA framework, assume that there are $n$ DMUs (DMU $j, j=1, \ldots, n)$ which consume $m$ inputs $\left(x_{i}, i=1, \ldots, m\right)$ to produce $s$ outputs $\left(y_{r}, r=1, \ldots, s\right)$. The first phase of CRA input-oriented model (CRA-I) developed by Lozano and Villa [15] measures the efficiency of system through solving the following linear program:

$$
\begin{array}{ll}
\min & \theta \\
\text { s.t. } & \sum_{k=1}^{n} \sum_{j=1}^{n} \lambda_{j k} x_{i j} \leq \theta \sum_{j=1}^{n} x_{i j} \quad i=1, \ldots, m \\
& \sum_{k=1}^{n} \sum_{j=1}^{n} \lambda_{j k} y_{r j} \geq \sum_{j=1}^{n} y_{r j} \quad r=1, \ldots, s \\
& \sum_{j=1}^{n} \lambda_{j k}=1 \quad k=1, \ldots, n \\
& \lambda_{j k} \geq 0 \quad k, j=1, \ldots, n .
\end{array}
$$

In Phase II of CRA model, an additional reduction of any inputs or expansion of any outputs is followed. Phase II is formulated to remove any possible input excesses and any output shortfalls as follows:

$$
\begin{array}{ll}
\max & \sum_{i=1}^{m} s_{i}^{-}+\sum_{r=1}^{s} t_{r}^{+} \\
\text {s.t. } & \sum_{k=1}^{n} \sum_{j=1}^{n} \lambda_{j k} x_{i j}+s_{i}^{-}=\theta^{*} \sum_{j=1}^{n} x_{i j} \quad i=1, \ldots, m \\
& \sum_{k=1}^{n} \sum_{j=1}^{n} \lambda_{j k} y_{r j}-t_{r}^{+}=\sum_{j=1}^{n} y_{r j} \quad r=1, \ldots, s \\
& \sum_{j=1}^{n} \lambda_{j k}=1 \quad k=1, \ldots, n \\
& s_{i}^{-} \geq 0, \quad t_{r}^{+} \geq 0 \quad i=1, \ldots, m, r=1, \ldots, s \\
& \lambda_{j k} \geq 0 \quad k, j=1, \ldots, n .
\end{array}
$$

Model (5) was formulated based on two important purposes. First, instead of reducing the inputs of each DMU, the aim is to reduce the total amount of input consumption of the DMUs. Second, after solving the problem in Phase II, the projection of all DMUs will be onto the efficient frontier of production possibility set. Indeed, the efficiency score of system is more important than efficiency score of each unit in the centralized scenario. For that reason, decision manager (DM) tries to reallocate resources to have a more efficient system. Toward this end, some of the inputs can be transferred from one DMU to other DMUs. It is not necessary to keep the total value of inputs or outputs in original level because the overall consumption may be decreased and the overall production may be increased. 
The improvement activity of $\mathrm{DMU}_{o}$, which is obtained by the maximum slack solution and is located on the efficiency frontier of production possibility set, is defined as follows:

$$
\begin{aligned}
& \overline{x_{i o}}=\sum_{j=1}^{n} \lambda_{j}^{o *} x_{i j}=\theta^{*} x_{i o}-s_{i}^{-*} \quad i=1, \ldots, m, \\
& \overline{y_{r o}}=\sum_{j=1}^{n} \lambda_{j}^{o *} y_{r j}=y_{r o}+t_{r}^{+*} \quad r=1, \ldots, s .
\end{aligned}
$$

The difference between the total consumption of improved activity and the original DMUs in each input and output can be found by the following relationship:

$$
\begin{aligned}
& S_{i}=\sum_{j=1}^{n} x_{i j}-\sum_{j=1}^{n} \overline{x_{i j}} \geq 0 \quad i=1, \ldots, m, \\
& T_{r}=\sum_{j=1}^{n} \overline{y_{r j}}-\sum_{j=1}^{n} y_{r j} \geq 0 \quad r=1, \ldots, s .
\end{aligned}
$$

The dual formulation of the envelopment form of the CRA input oriented model to find the common input and output weights, which maximize the relative efficiency score of a virtual DMU with the average inputs and outputs, can be written as follows:

$$
\begin{array}{ll}
\max & \sum_{j=1}^{n} \sum_{r=1}^{s} u_{r} y_{r j}+\sum_{k=1}^{n} \zeta_{k} \\
\text { s.t. } & \sum_{j=1}^{n} \sum_{i=1}^{m} v_{i} x_{i j}=1 \\
& \sum_{r=1}^{s} u_{r} y_{r j}-\sum_{i=1}^{m} v_{i} x_{i j}+\zeta_{k} \leq 0 \quad j, k=1, \ldots, n \\
& u_{r} \geq 0 \quad r=1, \ldots, s \\
& v_{i} \geq 0 \quad i=1, \ldots, m .
\end{array}
$$

The above model has $n^{2}+1$ constraints and $m+s+$ $n$ variables. Solving model (9) derives the common set of weights (CSW). It is worth mentioning that we can use this common set of weights to evaluate the absolute efficiency of each efficient DMU in order to rank them. The ranking adopts the CSW generated from model (9), which makes sense because a DM objectively chooses the common weights for the purpose of maximizing the group efficiency. For instance, the government is interested in measuring the performance of DEA efficient banks. The government would determine one common set of weights based upon the group performance of the DEA efficient banks.

\section{Proposed Method}

Proposing the model in this study, we used the distance directional function to measure the overall efficiency score of each system. Throughout this method, we deal with $n$ DMUs $(j=1, \ldots, n)$ having $m$ inputs $(i=1, \ldots, m)$ and $s$ outputs. The outputs are divided into two sets: one as desirable outputs and one as undesirable outputs. Let the inputs and desirable and undesirable outputs be as follows:

$$
\begin{aligned}
X & =\left\{x_{i j}\right\} \in R_{+}^{m \times n}, \quad Y^{D}=\left\{y_{r j}^{D}\right\} \in R_{+}^{s^{D} \times n}, \\
Y^{U} & =\left\{y_{t j}^{U}\right\} \in R_{+}^{s^{U} \times n},
\end{aligned}
$$

where $X, Y^{D}$, and $Y^{U}$ are input, desirable output, and undesirable output matrices, respectively. In our proposed model, we apply the distance directional function to reformulate the centralized resource allocation model when some outputs are undesirable. In addition, we consider undesirable outputs as inputs in evaluation. The model is as follows:

$$
\begin{array}{ll}
\max & \varphi \\
\text { s.t. } & \sum_{k=1}^{n} \sum_{j=1}^{n} \lambda_{j k} x_{i j} \leq \sum_{j=1}^{n} x_{i j}-\varphi R x_{i} \quad i=1, \ldots, m \\
& \sum_{k=1}^{n} \sum_{j=1}^{n} \lambda_{j k} y_{r j}^{D} \geq \sum_{j=1}^{n} y_{r j}^{D}+\varphi R y_{r}^{D} \quad r=1, \ldots, s^{D} \\
& \sum_{k=1}^{n} \sum_{j=1}^{n} \lambda_{j k} y_{t j}^{U} \leq \sum_{j=1}^{n} y_{r j}^{U}-\varphi R y_{t}^{U} \quad t=1, \ldots, s^{U} \\
& \sum_{j=1}^{n} \lambda_{j k}=1 \quad k=1, \ldots, n \\
& \lambda_{j k} \geq 0 \quad k, j=1, \ldots, n,
\end{array}
$$

where $R x_{i}, R y_{r}^{D}$, and $R y_{t}^{U}$ are parameters; also $s^{D}$ and $s^{U}$ stand for the number of desirable outputs and undesirable outputs, respectively. The objective of model (11) is to decrease inputs and undesirable outputs level and increase desirable outputs level with regard to the $\left(R x_{i}, R y_{r}^{D}, R y_{t}^{U}\right)$ direction. Here, we use the ideal point to assign to the $\left(R x_{i}, R y_{r}^{D}, R y_{t}^{U}\right)$ vector as follows:

$$
\begin{array}{cc}
R x_{i}=\sum_{j=1}^{n} x_{i j}-n\left(\min \left\{x_{i j}\right\}_{j=1, \ldots, n}\right) & i=1, \ldots, m, \\
R y_{r}^{D}=\sum_{j=1}^{n} y_{r j}^{D}-n\left(\max \left\{y_{r j}^{D}\right\}_{j=1, \ldots, n}\right) & r=1, \ldots, s^{D}, \\
R y_{t}^{U}=\sum_{j=1}^{n} y_{t j}^{U}-n\left(\min \left\{y_{t j}^{U}\right\}_{j=1, \ldots, n}\right) & t=1, \ldots, s^{U} .
\end{array}
$$

The optimal objective value of model (11) measures system inefficiency score. It is worth mentioning that another alternative for the directional vector $\left(R x_{i}, R y_{r}^{D}, R y_{t}^{U}\right)$ can be chosen as follows:

$$
\left(R x_{i}, R y_{r}^{D}, R y_{t}^{U}\right)=\left(\sum_{j=1}^{n} x_{i j}, \sum_{j=1}^{n} y_{r j}^{D}, \sum_{j=1}^{n} y_{t j}^{U}\right) .
$$

The purposes of model (11) are to reduce the total consumption of inputs, reduce the total production of undesirable 
TABLE 1: Data set with undesirable outputs.

\begin{tabular}{|c|c|c|c|c|c|c|}
\hline & \multicolumn{2}{|c|}{ Inputs } & \multicolumn{2}{|c|}{ Desirable outputs } & \multicolumn{2}{|c|}{ Undesirable outputs } \\
\hline & I1 & $\mathrm{I} 2$ & $\mathrm{O} 1$ & $\mathrm{O} 2$ & UO1 & $\mathrm{UO} 2$ \\
\hline DMU 1 & 5 & 8 & 9 & 15 & 4 & 3 \\
\hline DMU 2 & 7 & 5 & 12 & 19 & 9 & 7 \\
\hline DMU 3 & 5 & 4 & 18 & 21 & 4 & 3 \\
\hline DMU 4 & 6 & 8 & 14 & 11 & 10 & 6 \\
\hline DMU 5 & 7 & 7 & 11 & 14 & 8 & 8 \\
\hline DMU 6 & 8 & 3 & 10 & 17 & 4 & 9 \\
\hline DMU 7 & 5 & 5 & 16 & 10 & 6 & 5 \\
\hline DMU 8 & 4 & 9 & 19 & 9 & 5 & 2 \\
\hline Sum & 47 & 49 & 109 & 116 & 50 & 43 \\
\hline \multicolumn{7}{|c|}{ Projection points } \\
\hline DMU 1 & 5 & 8 & 9 & 15 & 4 & 3 \\
\hline DMU 2 & 7 & 5 & 12 & 19 & 9 & 7 \\
\hline DMU 3 & 5 & 4 & 18 & 21 & 4 & 3 \\
\hline DMU 4 & 6 & 8 & 14 & 11 & 10 & 6 \\
\hline DMU 5 & 7 & 7 & 11 & 14 & 8 & 8 \\
\hline DMU 6 & 8 & 3 & 10 & 17 & 4 & 9 \\
\hline DMU 7 & 5 & 5 & 16 & 10 & 6 & 5 \\
\hline DMU 8 & 4 & 9 & 19 & 9 & 5 & 2 \\
\hline Sum & 39.2 & 36 & 144.8 & 158.4 & 32.8 & 23.2 \\
\hline
\end{tabular}

TABLE 2: Current and optimized levels of the entire system.

\begin{tabular}{lcccccc}
\hline & \multicolumn{2}{c}{ Inputs } & \multicolumn{2}{c}{ Desirable outputs } & \multicolumn{2}{c}{ Undesirable outputs } \\
& $\mathrm{I} 1$ & $\mathrm{I} 2$ & $\mathrm{O} 1$ & $\mathrm{I} 1$ & $\mathrm{I} 2$ & 50 \\
Current level & 47 & 49 & 109 & 116 & 43 & 30 \\
Optimal level & 39.2 & 36 & 144.8 & 158.4 & 32.8 & 23.2 \\
Rate of reduction or increase & $16.5 \%$ & $26.5 \%$ & $24.7 \%$ & $26.7 \%$ & $34.4 \%$ & $46 \%$ \\
\hline
\end{tabular}

outputs, and increase the overall production of desirable outputs in the direction of $\left(R x_{i}, R y_{r}^{D}, R y_{t}^{U}\right)$, simultaneously. It should be pointed out that undesirable outputs are considered as inputs in the proposed model.

\section{Numerical Example}

To illustrate the proposed model (11), consider that a system consists of 8 DMUs and that each DMU consumes two inputs to produce four outputs (two desirable outputs and two undesirable outputs). Table 1 shows the data.

The efficiency score of the entire system can be readily obtained by using model (11), which is $48 \%$. Moreover, the projection points are shown in Table 1 . As can be seen from Table 2, we can compare the observed system with the projected system. For instance, model (11) suggests $16.5 \%$ and $26.5 \%$ saving (reduction) in the first and second inputs, respectively. In addition, by using model (11) to project all of DMUs onto the efficient frontier, DM could have $24.7 \%$ and $26.7 \%$ increases in producing the desirable output 1 and output 2 , respectively.

Increasing the production of desirable output 1 from 109 (current level) to 144.8 (optimum level) and increasing the production of desirable output 2 from 116 (current level) to
158.4 (optimum level) are meaningful. Model (11) also has a significant reduction plan in both undesirable outputs, that is, decreasing the production level of undesirable output 1 from 50 to 32.8 (34.4\% reduction) and decreasing the level of production of undesirable output 2 from 43 to 23.2 ( $46 \%$ reduction).

\section{Conclusion}

The issue of dealing with undesirable data in CRA is an important topic. The existing CRA models have been focused on desirable inputs and outputs. In this paper, we developed an approach proposed by Lozano and Villa [15] for dealing with undesirable outputs by using distance directional function. The CRA model presented here can be used for the analysis of any real situations where a significant number of desirable and undesirable outputs are included.

Moreover, the proposed model is able to suggest a managerial point of view to DM to make decision and come up with a plan for the system. In a similar way, the proposed model can be reformulated to deal with undesirable inputs' treatment in centralized resource allocation scenario. On the basis of the promising findings presented in this paper, work on the remaining issues is continuing and will be presented 
in future papers. Clearly, in our future research, we intend to concentrate on CRA model with imprecise, interval, and fuzzy data.

\section{Conflict of Interests}

The authors have no conflict of interests to disclose.

\section{References}

[1] A. Charnes, W. W. Cooper, and E. Rhodes, "Measuring the efficiency of decision making units," European Journal of Operational Research, vol. 2, no. 6, pp. 429-444, 1978.

[2] R. D. Banker, A. Charnes, and W. W. Cooper, "Some methods for estimating technical and scale inefficiencies in data envelopment analysis," Management Science, vol. 30, no. 9, pp. 10781092, 1984.

[3] A. Charnes, W. W. Cooper, B. Golany, L. Seiford, and J. Stutz, "Foundations of data envelopment analysis for ParetoKoopmans efficient empirical production functions," Journal of Econometrics, vol. 30, no. 1-2, pp. 91-107, 1985.

[4] K. Tone, "A slacks-based measure of efficiency in data envelopment analysis," European Journal of Operational Research, vol. 130, no. 3, pp. 498-509, 2001.

[5] K. Allen, "DEA in the ecological context an overview," in Data Envelopment Analysis in the Service Sector, G. Wesermann, Ed., pp. 203-235, Gabler, Wiesbaden, Germany, 1999.

[6] T. C. Koopman, "Analysis of production as an efficient combination of activities," in Activity Analysis of Production and Allocation, Cowles Commission, T. C. Koopmans, Ed., pp. 33-97, Wiley, New York, NY, USA, 1951.

[7] A. Iqbal Ali and L. M. Seiford, "Translation invariance in data envelopment analysis," Operations Research Letters, vol. 9, no. 6, pp. 403-405, 1990.

[8] J. T. Pastor, "Translation invariance in data envelopment analysis: a generalization," Annals of Operations Research, vol. 66, pp. 93-102, 1996.

[9] H. Scheel, "Undesirable outputs in efficiency valuations," European Journal of Operational Research, vol. 132, no. 2, pp. 400410, 2001.

[10] L. M. Seiford and J. Zhu, "Modeling undesirable factors in efficiency evaluation," European Journal of Operational Research, vol. 142, no. 1, pp. 16-20, 2002.

[11] B. Golany and Y. Roll, "An application procedure for DEA," Omega, vol. 17, no. 3, pp. 237-250, 1989.

[12] C. A. K. Lovell and J. T. Pastor, "Units invariant and translation invariant DEA models," Operations Research Letters, vol. 18, no. 3, pp. 147-151, 1995.

[13] H. Scheel, "Efficiency measurement system: DEA for windows," Software, Operations Research and Wirtschafts-informatik, Univeritat Dortmund, 1998,

[14] W. Liu and J. Sharp, "DEA models via goal programming," in Data Envelopment Analysis in the Service Sector, G. Westermann, Ed., pp. 79-101, Deutscher Universitatsverlag, Wiesbaden, Germany, 1999.

[15] S. Lozano and G. Villa, "Centralized resource allocation using data envelopment analysis," Journal of Productivity Analysis, vol. 22, no. 1-2, pp. 143-161, 2004.

[16] C. Mar-Molinero, D. Prior, M.-M. Segovia, and F. Portillo, "On centralized resource utilization and its reallocation by using DEA," Annals of Operations Research, 2012.
[17] P. Korhonen and M. Syrjänen, "Resource allocation based on efficiency analysis," Management Science, vol. 50, no. 8, pp. 11341144, 2004.

[18] J. Du, L. Liang, Y. Chen, and G. B. Bi, "DEA-based production planning," Omega, vol. 38, no. 1-2, pp. 105-112, 2010.

[19] M. Asmild, J. C. Paradi, and J. T. Pastor, "Centralized resource allocation BCC models," Omega, vol. 37, no. 1, pp. 40-49, 2009.

[20] F. Hosseinzadeh Lotfi, A. A. Noora, G. R. Jahanshahloo, J. Gerami, and M. R. Mozaffari, "Centralized resource allocation for enhanced Russell models," Journal of Computational and Applied Mathematics, vol. 235, no. 1, pp. 1-10, 2010.

[21] M.-M. Yu, C.-C. Chern, and B. Hsiao, "Human resource rightsizing using centralized data envelopment analysis: evidence from Taiwan's airports,' Omega, vol. 41, no. 1, pp. 119-130, 2013.

[22] A. Hadi Vencheh, R. Kazemi Matin, and M. Tavassoli Kajani, "Undesirable factors in efficiency measurement," Applied Mathematics and Computation, vol. 163, no. 2, pp. 547-552, 2005.

[23] G. R. Jahanshahloo, F. Hosseinzadeh Lotfi, N. Shoja, G. Tohidi, and S. Razavyan, "Undesirable inputs and outputs in DEA models," Applied Mathematics and Computation, vol. 169, no. 2, pp. 917-925, 2005.

[24] J. Wu and D. Guo, "A complete ranking of DMUs with undesirable outputs using restrictions in DEA models," Mathematical and Computer Modelling, vol. 58, no. 5-6, pp. 1102-1109, 2013.

[25] A. Amirteimoori, S. Kordrostami, and M. Sarparast, "Modeling undesirable factors in data envelopment analysis," Applied Mathematics and Computation, vol. 180, no. 2, pp. 444-452, 2006. 


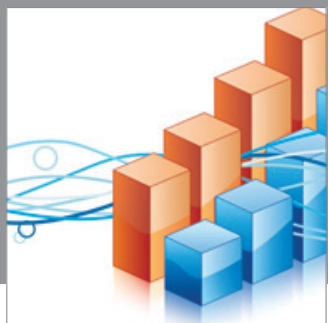

Advances in

Operations Research

mansans

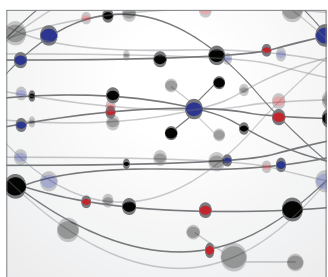

The Scientific World Journal
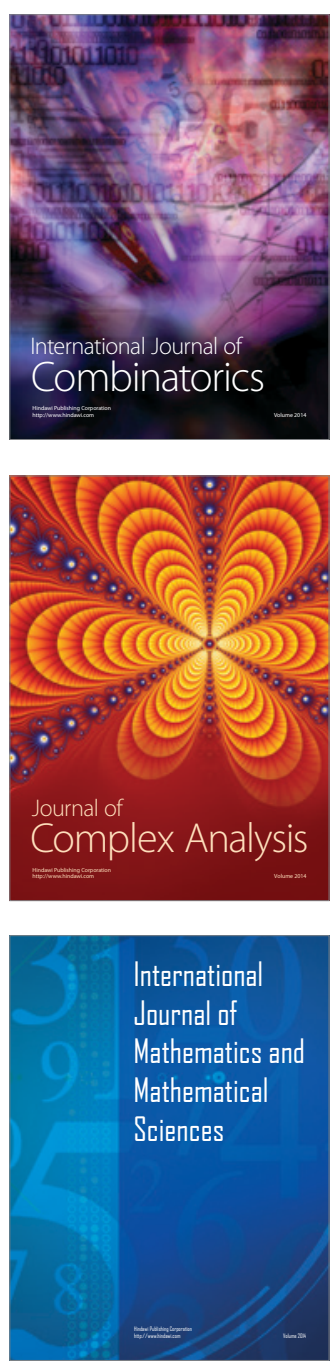
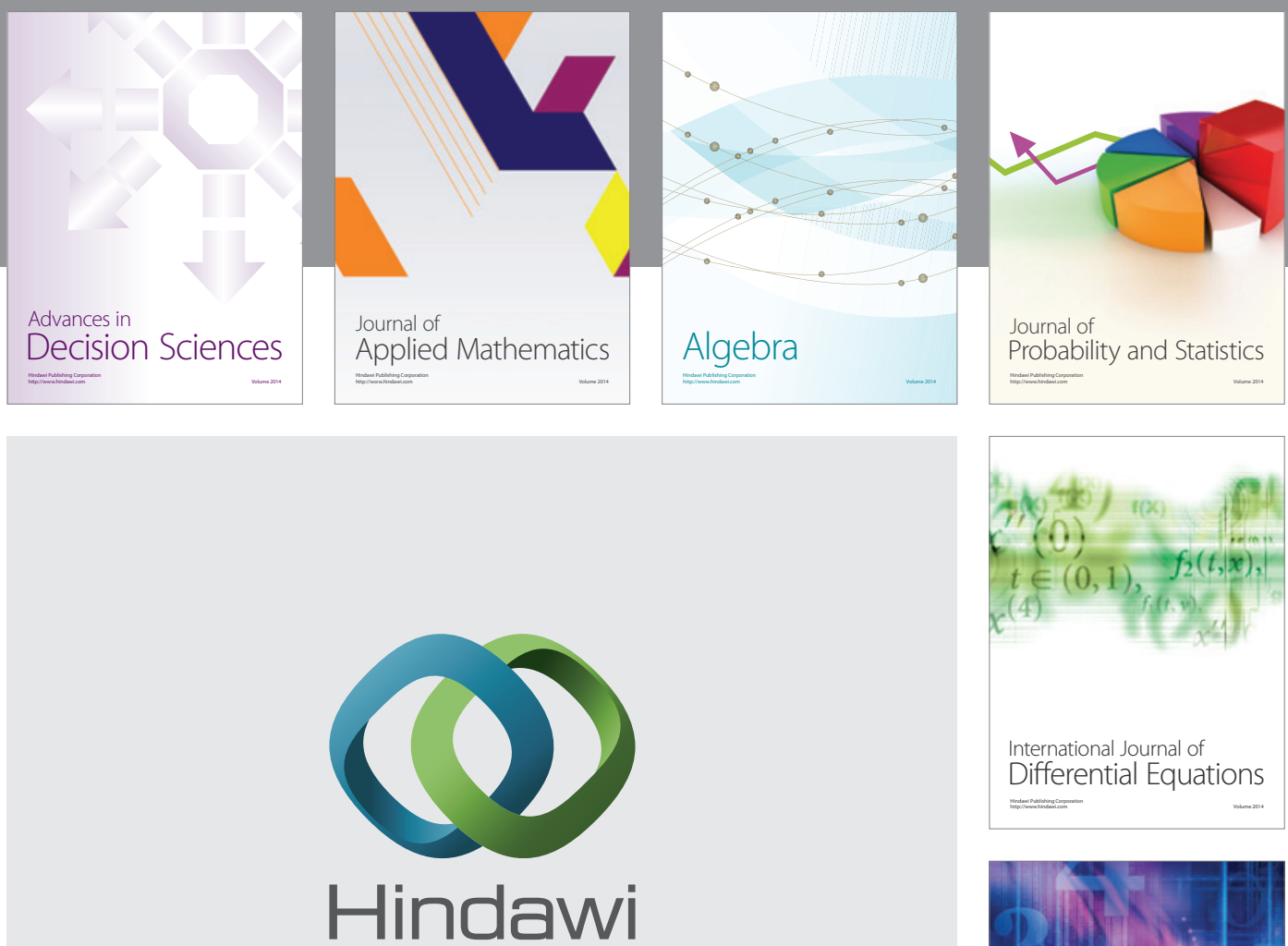

Submit your manuscripts at http://www.hindawi.com
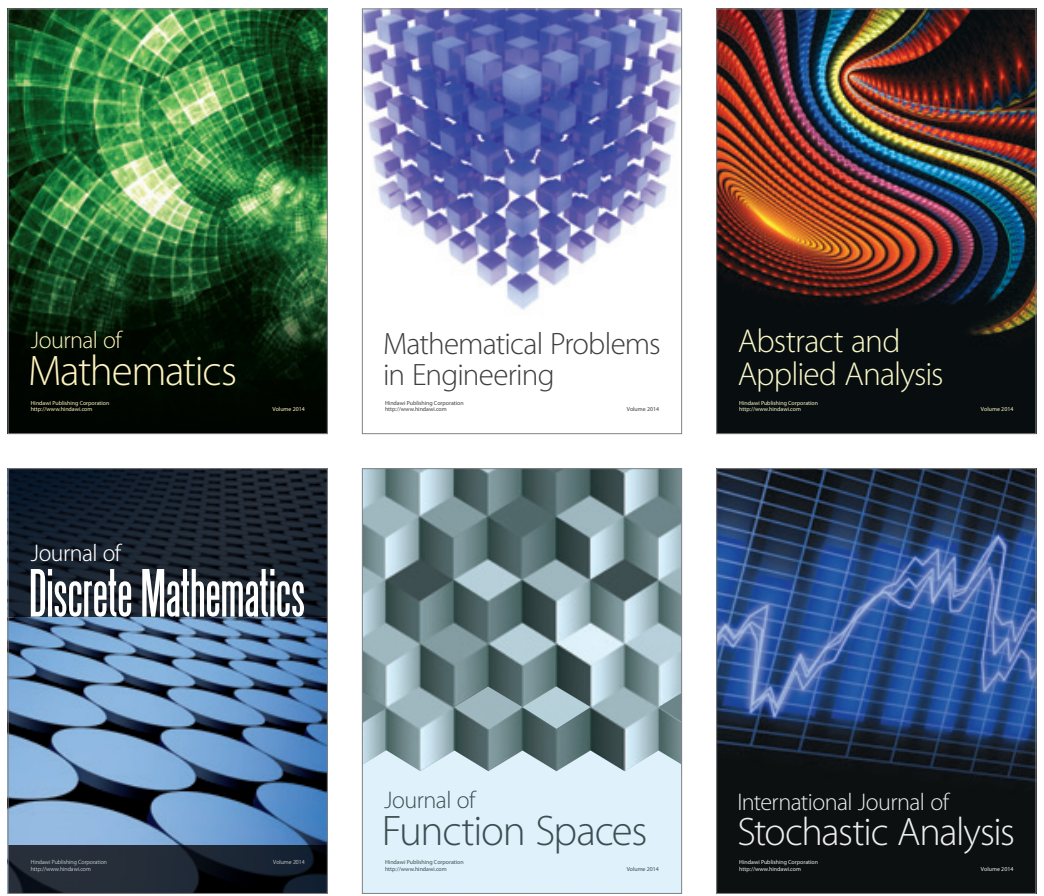

Journal of

Function Spaces

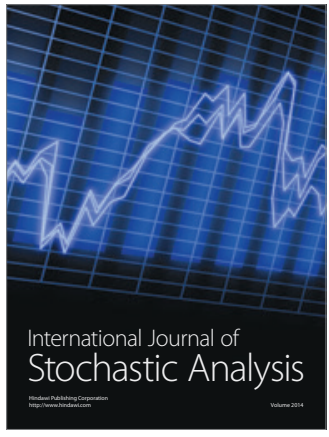

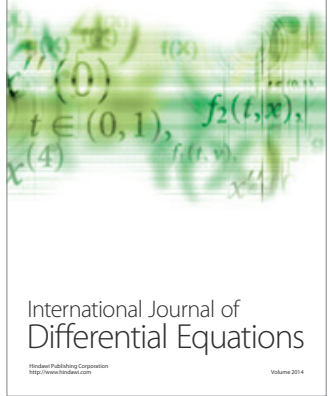
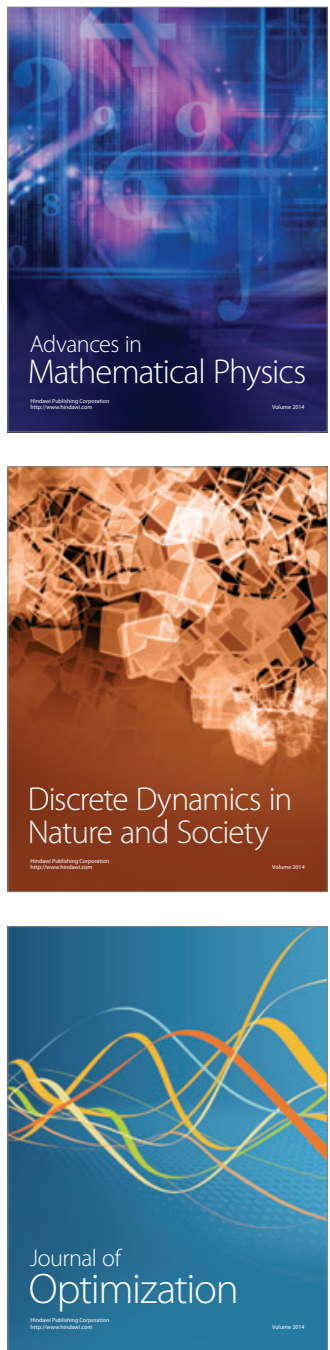